\title{
EXISTENCE OF NONNEGATIVE SOLUTIONS OF A SEMILINEAR EQUATION AT RESONANCE WITH LINEAR GROWTH
}

\author{
JAIRO SANTANILLA
}

(Communicated by Paul S. Muhly)

\begin{abstract}
A coincidence degree result is established to study sufficient conditions for the existence of nonnegative solutions of a semilinear equation at resonance in which the nonlinearity has at most linear growth. Nonnegative solutions to some boundary value problems are obtained to illustrate the theory.
\end{abstract}

The problem of existence of solutions in a convex set, or nonnegative solutions, for abstract semilinear equations at resonance has been recently considered by Nieto [20], Gaines and Santanilla [9], Mawhin and Rybakowski [19], and Santanilla [22]. They have considered the problem of existence of solutions to

$$
L u=N u
$$

in a convex set, where $L$ : $\operatorname{dom} L \subset X \rightarrow Z$ is a Fredholm operator of index zero, $N: X \rightarrow Z$ is not necessarily linear and satisfies a compactness property relative to $L$, and $X, Z$ are real Banach spaces. Using the alternative method, Nieto [20] introduced sufficient conditions for the existence of solutions to Equation (1) in a cone, when the nonlinearity $N$ is bounded. In this paper we shall use coincidence degree $[8,18]$ to present an extension of Nieto's result when $N$ grows linearly and $C$ is a wedge. Our result implies the Granas fixed point theorem and some results of Cesari and Kannan $[3,6]$ which have been extensively used in differential equations $[3,4,5,7,15,16]$. We shall also apply our abstract results to discuss the existence of nonnegative solutions to some boundary value problems when the nonlinearity is a Carathéodory function and has at most linear growth.

Notation and Definitions. Throughout this paper we shall assume that $L$ is a linear Fredholm operator of index zero; i.e. the image of $L, \operatorname{im} L$, is closed

Received by the editors December 14, 1987.

1980 Mathematics Subject Classification (1985 Revision). Primary 47H15, 34B15, 34C15, $35 \mathrm{G} 30,35 \mathrm{~J} 40$.

Key words and phrases. Coincidence degree, semilinear equations at resonance, boundary value problems. 
in $Z$ and $\operatorname{dim}(\operatorname{ker} L)=\operatorname{codim}(\operatorname{im} L)<\infty$. It follows from standard results of linear functional analysis that there exist continuous projectors $P: X \rightarrow X$ and $Q: Z \rightarrow Z$ such that $\operatorname{im} P=\operatorname{ker} L$ and $\operatorname{ker} Q=\operatorname{im} L$. We denote by $K_{p}: \operatorname{im} L \rightarrow \operatorname{dom} L \cap \operatorname{ker} P$ the partial inverse of $L$ and by $J$ an isomorphism from $\operatorname{im} Q$ onto $\operatorname{ker} L$. We assume that $0<\left\|K_{p}(I-Q)\right\|<\infty$.

Let $\Omega \subset X$ be an open bounded subset of $X$. We assume that $N: \bar{\Omega} \rightarrow Z$ is $L$-compact on $\bar{\Omega}$; i.e. $Q N: \bar{\Omega} \rightarrow Z$ and $K_{p}(I-Q) N: \bar{\Omega} \rightarrow X$ are compact on $\bar{\Omega}$. The reader is referred to $[8,18]$ for a complete discussion of coincidence degree.

Suppose there exists a continuous bilinear form on $Z \times X$

$$
(z, x) \mapsto\langle z, x\rangle
$$

such that

$$
z \in \operatorname{im} L \text { if and only if }\left\langle z, u_{0}\right\rangle=0
$$

for every $u_{0} \in \operatorname{ker} L$. Thus, if $\left\{v_{1}, v_{2}, \ldots, v_{n}\right\}$ is a basis of $\operatorname{ker} L$, then

$$
\begin{aligned}
& J: \operatorname{im} Q \rightarrow \operatorname{ker} L \\
& z \mapsto \sum_{i=1}^{n}\left\langle z, v_{i}\right\rangle v_{i}
\end{aligned}
$$

is an isomorphism and $\left\langle J^{-1} u_{0}, u_{0}\right\rangle>0$ if $u_{0} \neq 0$.

Finally, $C$ will denote a wedge in $X$; i.e. $C$ is a nonempty closed convex subset of $X$ such that $\alpha C \subset C$ for all $\alpha \geq 0$. We shall assume that there exists a continuous retraction $\gamma: X \rightarrow C$ of $C$, mapping bounded sets into bounded sets.

Theorem 1. Assume that the following conditions are satisfied.

(i) There exists constants $c_{1}>0$ and $0 \leq c_{2}<\left\|K_{p}(I-Q)\right\|^{-1}$ such that

$$
\|N u\| \leq c_{1}+c_{2}\|u\|
$$

for all $u \in C$.

(ii) There exists $R>0$ such that

$$
\left\langle Q N\left(u_{0}+u_{1}\right), u_{0}\right\rangle \leq 0
$$

for all $u=u_{0}+u_{1} \in C$, where $u_{0} \in \operatorname{ker} L,\left\|u_{0}\right\|=R, u_{1} \in \operatorname{ker} P$, and

$$
\left\|u_{1}\right\| \leq \rho \equiv\left(c_{1}+c_{2} R\right) /\left(\left\|K_{p}(I-Q)\right\|^{-1}-c_{2}\right) .
$$

(iii) $\left(P+J Q N+K_{p}(I-Q) N\right) \gamma(\bar{\Omega}) \subset C$, where

$$
\Omega=\left\{u=u_{0}+u_{1} \in X:\left\|u_{0}\right\|<R,\left\|u_{1}\right\|<\rho\right\} .
$$

Then $L u=N u$ has at least one solution $u \in \bar{\Omega} \cap C$.

Proof. Consider the homotopy

$$
M(u, \lambda) \equiv \lambda\left(P+J Q N+K_{p}(I-Q) N\right) \gamma u, \quad \lambda \in[0,1], u \in \bar{\Omega} .
$$


If $u=M(u, \lambda)$ for some $u \in \partial \Omega$ and some $\lambda \in(0,1)$, then $u \in C$ and

$$
u=\lambda\left(P u+J Q N u+K_{p}(I-Q) N\right) u .
$$

Thus,

$$
u_{0}=\lambda(P u+J Q N u), \quad u_{1}=\lambda K_{p}(I-Q) N u .
$$

If $\left\|u_{0}\right\|=R$ and $\left\|u_{1}\right\| \leq \rho$, we have

$$
0<(1-\lambda)\left\langle J^{-1} u_{0}, u_{0}\right\rangle=\lambda\left\langle Q N u, u_{0}\right\rangle \leq 0,
$$

a contradiction.

If $\left\|u_{0}\right\| \leq R$ and $\left\|u_{1}\right\|=\rho$, we obtain

$$
\begin{aligned}
\rho & =\left\|u_{1}\right\|=\lambda\left\|K_{p}(I-Q) N u\right\| \\
& \leq \lambda\left\|K_{p}(I-Q)\right\|\left(c_{1}+c_{2}\|u\|\right) \\
& \leq \lambda\left\|K_{p}(I-Q)\right\|\left(c_{1}+c_{2} R+c_{2} \rho\right) \\
& <c_{2}\left\|K_{p}(I-Q)\right\| \rho+\left(c_{1}+c_{2} R\right)\left\|K_{p}(I-Q)\right\|=\rho,
\end{aligned}
$$

another contradiction. By the invariance under homotopy property of the LeraySchauder degree, there exists $u \in \bar{\Omega}$ such that

$$
u=M(u, 1) \in C .
$$

The result follows from the fact that fixed points of $P+J Q N+K(I-Q) N$ are solutions of $L u=N u$.

Remarks. 1. When $c_{2}=0$, our result implies that of Nieto [20] which in turns generalizes results in $[6,24]$.

2. Our theorem is a partial extension of a result by Cesari and Kannan [3, Corollary 3] where $C=X$ is a Hilbert space, $\|N u\| \leq c_{1}+c_{2}\|u\|^{k}$ with $k \geq 1$ and $c_{1}$ is sufficiently small.

3. Similar results have been obtained by Gaines and Mawhin [8, Chapter VII] and Kannan and V. Lakshmikantham [13] when $C=X$.

4. The results in $[9,19,22]$ do not assume (explicitly) any growth condition on the nonlinearity. However, they require the evaluation of a Brouwer degree.

5. The inequality (2) can be reversed by replacing $J$ by $-J$ (in (iii)).

6. If $\operatorname{ker} L=\{0\}$ it follows that $P=Q=0$ and Theorem 1 reduces to

Corollary 2. Suppose that $\operatorname{ker} L=\{0\}, K_{0} N(C) \subset C$ and there exist constants $c_{1}>0$ and $c_{2} \geq 0$ with $c_{2}<\left\|K_{0}\right\|^{-1}$ such that

$$
\|N u\| \leq c_{1}+c_{2}\|u\|
$$

for all $u \in C$. Then $L u=N u$ has a solution in $C$.

This Corollary, which will be useful in the next section, is an extension of the classical Granas fixed point theorem. 
Corollary 3 (Granas [11]). If $N: X \rightarrow X$ is a completely continuous mapping in $a$ Banach space $X$ and there exist two constants $c_{1}>0$ and $0 \leq c_{2}<1$ such that $\|N u\| \leq c_{1}+c_{2}\|u\|$, for all $u \in X$, then $N$ has at least one fixed point. Proof. Take $X=Z, L=I$ and $C=X$ in Corollary 2.

The techniques in the proof of Theorem 1 may be used to obtain a version of Corollary 2 without the same growth condition on the nonlinearity.

Proposition 4. Let $\operatorname{ker} L=\{0\}$ and suppose there exists $\rho>0$ such that

$$
\left\|K_{0} N u\right\| \leq \rho
$$

for all $u \in C$ with $\|u\|=\rho$,

$$
K_{0} N \gamma u \in C
$$

for all $u \in X$ with $\|u\| \leq \rho$. Then $L u=N u$ has a solution $u$ in $C$ with $\|u\| \leq \rho$.

Proof. Consider the homotopy $M(u, \lambda)=\lambda K_{0} N \gamma u, \lambda \in[0,1]$ and $\|u\| \leq \rho$. Suppose

$$
u=\lambda K_{0} N \gamma u
$$

for some $\lambda \in(0,1)$ and some $u \in X$ with $\|u\|=\rho$. We have

$$
\rho=\|u\| \leq \lambda\left\|K_{0} N u\right\| \leq \lambda \rho<\rho,
$$

a contradiction. The result follows from the Leray-Schauder continuation theorem.

Note that Proposition 4 reduces to Rothe's fixed point theorem [21] when $X=Z, L=I$ and $C=X$. For another extension of Rothe's fixed point theorem, via degree theory, the reader is referred to [18]. Granas' theorem has also been extended in [1].

Examples. We shall apply Theorem 1 to obtain nonnegative solutions to

$$
\begin{gathered}
u^{\prime \prime}=f(x, u)=0, \quad \text { a.e. on } I=[0,1], \\
u(0)-u(1)=u^{\prime}(0)-u^{\prime}(1)=0
\end{gathered}
$$

where $f: I \times \mathbf{R} \rightarrow \mathbf{R}$ satisfies the Caratheodory condition; i.e. $f(x, \cdot)$ is continuous for a.e. $x \in I$ and $f(\cdot, u)$ is (Lebesgue) measurable for each $u \in \mathbf{R}$. We shall assume that $f$ has at most linear growth; i.e. there exist $c_{1} \in L^{2}(I)$ and $c_{2} \geq 0$ such that

$$
|f(x, u)| \leq c_{1}(x)+c_{2}|u|
$$

for a.e. $x \in I$ and all $u \in \mathbf{R}$.

Let $X=Z=L^{2}(I)$ with the usual norm. Define $\operatorname{dom} L=\{u:[0,1] \rightarrow \mathbf{R}$; $u, u^{\prime}$ are absolutely continuous, $u^{\prime \prime} \in L^{2}(I)$ and $u(0)-u(1)=u^{\prime}(0)-u^{\prime}(1)=$ $0\}, L u=-u^{\prime \prime}$. Thus $L$ is a Fredholm operator of index zero with

$\operatorname{ker} L=\langle 1\rangle$ 
and compact generalized inverse $K_{p}: \operatorname{im} L \rightarrow \operatorname{dom} L \cap \operatorname{ker} P$ (given below), where the orthogonal projection $P: X \rightarrow \operatorname{ker} L$ is given by

$$
(P u)(x)=\int_{0}^{1} u(s) d s, \quad x \in I .
$$

The operator

$$
N: L^{2} \rightarrow L^{2}, u \rightarrow-f(\cdot, u(\cdot)),
$$

is $L$-compact on bounded subsets of $L^{2}(I)$ and problem (3) is equivalent to $L u=N u$. Our task is to show that this equation has a solution in $C=\{u \in$ $L^{2}(I): u(x) \geq 0$ a.e. on $\left.I\right\}$.

Theorem 5. Suppose that $f$ has at most linear growth and there exist constants $\alpha \in(0,8], a, b>0$ such that

$$
-a+b u \leq f(x, u) \leq \alpha u
$$

for a.e. $x \in I$ and all $u \geq 0$, then (3) has a nonnegative solution.

Proof. It can be shown that for each $z \in L^{2}(I)$

$$
K_{p}(I-Q) z \equiv K_{p}(I-P) z=\sum_{k=1}^{\infty} \frac{\phi_{k}}{\lambda_{k}} \int_{0}^{1} z(x) \phi_{k}(x) d x,
$$

where

$$
\lambda_{k}=4 \pi^{2} k^{2}, \quad k=1,2, \ldots,
$$

are the eigenvalues of $L$ with corresponding eigenfunctions $\phi_{k}$. Thus

$$
\left\|K_{p}(I-P)\right\|=\sup _{k \geq 1} \frac{1}{\lambda_{k}}=\frac{1}{\lambda_{1}}=\frac{1}{4 \pi^{2}}
$$

and condition (i) of Theorem 1 is fulfilled with $c_{1}=a$ and $c_{2}=\alpha$.

Let $\beta>0$ (to be determined below). If we define the bilinear form $\langle$,$\rangle by$

$$
\langle u, z\rangle=\beta \int_{0}^{1} u(x) z(x) d x,
$$

then we may take $J=\beta I$ where $I$ is the identity mapping, and condition (ii) is obtained from the first inequality in (5), as follows. Let $u(x) \geq 0$ a.e. on $I, u(x)=R+u_{1}(x)$, where $R=a / b$ and

$$
\int_{0}^{1} u_{1}(x) d x=0
$$

Then,

$$
\left\langle P N u, u_{0}\right\rangle=-\beta R \int_{0}^{1} f(x, u(x)) d x \leq-\beta R\left(a-b \int_{0}^{1} u(x) d x\right)=0 .
$$

For each $z \in \operatorname{im} L$, 


$$
\left(K_{p} z\right)(x)=\int_{0}^{1} G(s, x) z(s) d s
$$

where

$$
G(s, x)=\frac{1}{2} \begin{cases}s(s+1-2 x), & 0 \leq s<x \leq 1 \\ (1-s)(2 x-s), & 0 \leq x \leq s \leq 1\end{cases}
$$

and

$$
\left(K_{p}(I-P) z\right)(x)=\int_{0}^{1} G_{2}(s, x) z(s) d s,
$$

where

$$
G_{2}(s, x)= \begin{cases}\frac{s^{2}+s-2 s x}{2}+\frac{x^{2}}{2}-\frac{x}{2}+\frac{1}{12}, & 0 \leq s<x \leq 1, \\ \frac{s^{2}-1 s-2 s x}{2}+\frac{x^{2}}{2}+\frac{x}{2}+\frac{1}{12}, & 0 \leq x \leq s \leq 1 .\end{cases}
$$

Define $\gamma: L^{2}(I) \rightarrow C$ by

$$
(\gamma u)(x)=|u(x)|
$$

and let us denote $(\gamma u)(x) \equiv \gamma_{u}(x)$.

$$
\begin{aligned}
P \gamma_{u}(x)+ & J Q N \gamma_{u}(x)+K_{p}(I-Q) N \gamma_{u}(x) \\
= & \int_{0}^{1} \gamma_{u}(s) d s-\beta \int_{0}^{1} f\left(s, \gamma_{u}(s)\right) d s \\
& -\int_{0}^{1} G_{2}(s, x) f\left(s, \gamma_{u}(s) d s\right. \\
& =\int_{0}^{1} \gamma_{u}(s) d s+\int_{0}^{1}\left[-\beta-G_{2}(s, x)\right] f\left(s, \gamma_{u}(s)\right) d s .
\end{aligned}
$$

Taking $\beta=\frac{1}{24}$ it can be shown that $-\frac{1}{8} \leq-\beta-G_{2}(s, x) \leq 0$. From the second inequality in (5) we have,

$$
P \gamma_{u}(x)+J Q N \gamma_{u}(x)+K_{p}(I-Q) N \gamma_{u}(x) \geq\left(1-\frac{\alpha}{8}\right) \int_{0}^{1} \gamma_{u}(s) d s \geq 0 .
$$

Thus, condition (iii) of Theorem 1 is fulfilled and the proof is complete.

The operator $L$ associated to problem (3) admits a decomposition of the form $T T^{*}$ where $T$ and its adjoint $T^{*}$ are the differential operators given by

$$
\operatorname{dom} T=\left\{u \in H^{1}(I): u(0)=u(1)\right\}, \quad T u=u^{\prime}
$$

and

$$
\operatorname{dom} T^{*}=\left\{u \in H^{\prime}(I): u(0)=u(1)\right\}, \quad T^{*} u=-u^{\prime} .
$$

$T^{*}$ is a Fredholm operator of index zero with

$$
\operatorname{ker} T^{*}=\langle 1\rangle
$$


and compact generalized inverse $K_{p}^{*}$ (given below), where $P$ is the orthogonal projector considered above. $N$ is $T^{*}$-compact and the problem of finding nonnegative solutions to

$$
u^{\prime}=f(x, u), \quad u(0)=u(1)
$$

is equivalent to solving the equation $T^{*} u=N u$ in $C$.

Theorem 6. Suppose that $f$ has at most linear growth and there exist constants $a>0$ and $b \in(0,1]$ such that

$$
-a+b u \leq f(x, u) \leq u
$$

for a.e. $x \in I$ and all $u \geq 0$. Then (6) has a nonnegative solution.

Proof. It follows from a result by Kannan and Locker [14, p. 70] that

$$
\left\|K_{p}^{*}(I-P)\right\|=\sqrt{\left\|K_{p}(I-P)\right\|}=\frac{1}{2 \pi} .
$$

Thus, (i) of Theorem 1 is fulfilled with $c_{1}=a$ and $c_{2}=1$. Condition (ii) follows as in the proof of Theorem 5 .

It can be shown that for each $z \in \operatorname{im} L$,

$$
\left(K_{p}^{*} z\right)(x)=\int_{0}^{1} \tilde{G}(s, x) z(s) d s,
$$

where

$$
\widetilde{G}(s, x)= \begin{cases}-(s+1), & 0 \leq s<x \leq 1 \\ -s, & 0 \leq x \leq s \leq 1\end{cases}
$$

and

where

$$
\left(K_{p}^{*}(I-P) z\right)(x)=\int_{0}^{1} G_{1}(s, x) z(s) d s,
$$

$$
G_{1}(s, x)= \begin{cases}x-s-\frac{1}{2}, & 0 \leq s<x \leq 1, \\ x-s+\frac{1}{2}, & 0 \leq x \leq s \leq 1 .\end{cases}
$$

Using the retraction $\gamma$ defined above, we obtain

$$
\begin{aligned}
& P \gamma_{u}(x)+J Q N \gamma_{u}(x)+K_{p}^{*}(I-Q) N \gamma_{u}(x) \\
& =\int_{0}^{1} \gamma_{u}(s) d s-\beta \int_{0}^{1} f\left(s, \gamma_{u}(s)\right) d s \\
& \quad-\int_{0}^{1} G_{1}(s, x) f\left(s, \gamma_{u}(s)\right) d s \\
& \quad=\int_{0}^{1} \gamma_{u}(s) d s+\int_{0}^{1}\left[-\beta-G_{1}(s, x)\right] f\left(s, \gamma_{u}(s)\right) d s .
\end{aligned}
$$

If $\beta=\frac{1}{2}$ it follows that $-1 \leq-\beta-G_{1}(s, x) \leq 0$ and, assumption (iii) of Theorem 1 follows from the second inequality in (7) and the proof is complete.

According to our abstract result the nonnegative solutions to (3) and (6) are bounded in the $L^{2}$-norm. For instance, a solution $u$ given by Theorem 5 satisfies $|u|_{2} \leq\left(a+\alpha a b^{-1}\right) /\left(4 \pi^{2}-\alpha\right)+a / b$. 
Finally we shall mention that coincidence degree has been used by Gaines and Santanilla [9] and the author [22, 23] to obtain nonnegative periodic solutions to systems of first and second order ordinary differential equations when the nonlinearity is continuous. In contrast to the results presented here, the arguments to obtain a priori bounds in those papers heavily depend upon the continuity of $f$ on the first variable. For additional results on the subject, we mention the references in $[9,12,17,22,23]$.

Finally we shall obtain nonnegative solutions to

$$
\begin{gathered}
u^{\prime \prime}+f(x, u)=0, \quad \text { a.e. on }[0, \pi] \\
u(0)=u(\pi)=0
\end{gathered}
$$

where $f:[0, \pi] \times \mathbf{R} \rightarrow \mathbf{R}$ is a Carathéodory function having at most linear growth. We have

Theorem 7. Suppose there exist constants $c_{1}>0$ and $0 \leq c_{2}<1$ such that

$$
0 \leq f(x, u) \leq c_{1}+c_{2} u
$$

for a.e. $x \in[0, \pi]$ and all $u \geq 0$. Then (7) has a nonnegative solution.

Proof. With obvious modifications, we use the notation associated to problem (3) with $N u=f(\cdot, u(\cdot))$ and apply Corollary 2. Clearly $\operatorname{ker} L=\{0\}$ and the inverse $K_{0}$ of $L, L u=-u^{\prime \prime}$, is given by

$$
\left(K_{0} z\right)(x)=\int_{0}^{\pi} G(x, s) z(s) d s
$$

where

$$
G(x, s)=\frac{1}{\pi} \begin{cases}(\pi-s) x, & 0 \leq x \leq s \leq \pi \\ (\pi-x) s, & 0 \leq s \leq x \leq \pi\end{cases}
$$

The first nonzero eigenvalue of $-u^{\prime \prime}-\lambda u=0, u(0)=u(\pi)=0$ is $\lambda_{1}=1$. Thus, $\left\|K_{0}\right\|=1 / \lambda_{1}=1$. The result follows from Corollary 2 .

When the nonlinearity $f$ is at least continuous, nonnegative solutions to (7) have been studied in $[2,10,12,17,22,23]$.

\section{REFERENCES}

1. A. Cañada and P. Martinez-Amores, Solvability of some operator equations and periodic solutions of nonlinear functional differential equations, J. Differential Equations 49 (1983), $415-$ 429.

2. A. Castro and R. Shivaji, Nonnegative solution for a class of nonpositone problems, preprint.

3. L. Cesari and R. Kannan, Solutions of nonlinear hyperbolic equations at resonance, Nonlinear Analysis, TMA 6 (1982), 751-805.

4. L. Cesari, R. Kannan and J. Schuur Edit., Nonlinear functional analysis and differential equations, Dekker, New York, 1976.

5. L. Cesari and R. Kannan, Qualitative study of a class of nonlinear boundary value problems at resonance, J. Differential Equations 56 (1985), 63-81.

6. __, An abstract theorem at resonance, Proc. Amer. Math. Soc. 63 (1977), 221-225. 
7. __ Existence of solutions of a nonlinear differential equation, Proc. Amer. Math. Soc. 4 (1983), 605-613.

8. R. E. Gaines and J. Mawhin, Coincidence degree and nonlinear differential equations, Lecture Notes in Math., vol. 568, Springer-Verlag, Berlin, 1977.

9. R. Gaines and J. Santanilla, $A$ coincidence theorem in convex sets with applications to periodic soltuions of ordinary differential equations, Rocky Mountain J. Math. 12 (1982), 669-678.

10. J. A. Gatica and H. Smith, Fixed point techniques in a cone with application, J. Math. Anal. Appl. 61 (1977), 58-71.

11. A. Granas, On a certain class of nonlinear mappings in Banach spaces, Bull. Acad. Polon. Sci. Ser. Sci. Math. Astronom. Phys. 9 (1957), 867-871.

12. G. B. Gustafson and K. Schmitt, Nonzero solutions of boundary value problems for second order ordinary and delay-differential equations, J. Math. Anal. Appl. 12 (1972), 129-147.

13. R. Kannan and V. Lakshmikantham, Periodic solutions of nonlinear boundary value problems, Nonlinear Analysis, TMA 6 (1982), 1-10.

14. R. Kannan and J. Locker, Nonlinear boundary value problems and operators $T T^{*}$, J. Differential Equations 28 (1978), 60-103.

15. R. Kannan, J. J. Nieto and M. B. Ray, A class of nonlinear boundary value problems without Landesman-Lazer condition, J. Math. Anal. Appl. 105 (1985), 1-11.

16. R. Kannan, J. J. Nieto and V. Lakshmikantham, Sufficient conditions for existence of solutions of nonlinear boundary value problems at renonance, Nonlinear Anal. TMA 7 (1983), 10131020.

17. M. A. Krasnosel ' skii, Positive solutions of operator equations, Noordhoff, Groningen, 1964.

18. J. Mawhin, Topological degree methods in nonlinear boundary value problems, CBMS Regional Conference Series in Math. no. 40, Amer. Math. Soc., Providence, R.I. 1979, 1981.

19. J. Mawhin and K. P. Rybakowski, Continuation theorems for semi-linear equations in Banach spaces, preprint.

20. J. Nieto, Existence of solutions in a convex set for nonlinear alternative problems, Proc. Amer. Math. Soc. 94 (1985), 433-436.

21. E. Rothe, Zur Theorie der topologischer Ordnung und dere Vektorfelder in Banachsen Raumen, Compositio Math. 5 (1937), 177-197.

22. J. Santanilla, Some coincidence theorem in wedges, cones and convex sets, J. Math. Anal. Appl. 105 (1985), 357-371.

23. __ Nonnegative solutions to boundary value problems for nonlinear first and second order ordinary differential equations, J. Math. Anal. Appl. 126 (1987), 397-408.

24. J. Mawhin, Landesman-Lazer's type problems for nonlinear equations, Conf. Sem. Mat. Univ. Bari, (1977), No. 147.

Department of Mathematics, University of New Orleans, New Orleans, Louisiana 70148 